\title{
Analysis on the Winding Process of Pre-stressed Wire Wound Orthogonal Preload Frame on the Basis of Finite Element
}

\author{
Wenjie Wang ${ }^{1, a^{*}}$, Feng Lin ${ }^{1, b}$, Lei Zhang ${ }^{1, c}$, Xin Wang ${ }^{1, d}$ \\ ${ }^{1}$ Department of Mechanical Engineering, Tsinghua University, Beijing, 100084, China \\ awangwenjiedlut@163.com, blinfeng@tsinghua.edu.cn, 'stoneszhang@mail.tsinghua.edu.cn, \\ dwangxin-11@163.com
}

Keywords: Pre-stressed, orthogonal preload frame, steel wire winding, finite element, temperature drop method

\begin{abstract}
During the winding construction, the pre-stressed wire wound orthogonal preload frame bears the preloading force applied by the steel wire from top, bottom, left and right simultaneously. Compared with the ordinary preload frame, it bears complex force, there is no obvious distribution rule on the surface stress field, and it is difficult to monitor the preloading force. The monitoring of preloading force is crucial for the wire wound process and will directly influence the working performance and safety during the manufacturing process of multi-ram die forging hydraulic press. It uses the finite element wire layer temperature drop method to simulate the winding process of pre-stressed wire wound orthogonal preload frame, studies the distribution of surface stress field during the winding process, confirms its unidirectional stress distribution area and key points of stress monitoring and analyzes the overall deformation during its winding process, so as to solve the pre-stressed monitoring problem and overall stiffness analysis during the winding process. The result demonstrates that the finite element computation result of overall deformation of frame substantially agrees with the test result, and it shows the feasibility of simulating the steel wire winding process by temperature drop method. In addition, the result of preloading force measurement based on the sticking scheme established by finite element substantially agrees with the theoretical value of design, and it solves the pre-stressed monitoring problem during the process of winding construction of pre-stressed wire wound frame.
\end{abstract}

\section{Introduction}

Multi-ram die forging technology is a die forging-extrusion process and is widely applied to petroleum, aviation and electric power industries ${ }^{[1]}$, the precise forging parts and tools produced by this technology have a series of advantages of easy control of metal flow direction, continuous metal flow line, no-fin and saving metal ${ }^{[2]}$, and it is an advanced, precise, energy-saving and material-saving green manufacturing technology ${ }^{[3-4]}$. However, the multi-ram die forging hydraulic presses over 1000-ton in the world mainly include three multi-ram die forging hydraulic presses of $100 \mathrm{MN}, 180 \mathrm{MN}$ and $300 \mathrm{MN}$ manufactured by American Cameron Company respectively in 1952, 1961 and 1967 and one 100 MN multi-ram die forging press produced by China in 1970s ${ }^{[5-7]}$. The reason of such current situation is that the design of the bearing structure of heavy multi-ram die forging press has the core problem that the structural independence and the mechanical independence could not be satisfied simultaneously ${ }^{[8]}$. In consideration of the characteristics of the pre-stressed wire-wound structure of great bearing capacity, high fatigue strength and the like ${ }^{[9]}$, it proposes the pre-stressed wire-wound orthogonal preload frame, referred to as PWOPF, as shown in figure 1. It means winding the external profile of overall frame composed by multidirectional arc and straight line by high-tensile steel wires, generating the preloading force corresponding to the multidirectional pressing load direction and balancing the stress produced by the multidirectional load.

PWOPF is applied to heavy multi-ram die forging hydraulic press. For the convenience of transportation and processing, PWOPF is partitioned along the normal direction of the outer contour at the junction of linear segment and the arc segment, it uses the micro-bumpy-ridge surface between 
partition sub-components and the winding preloading force by tons of steel wires to keep its integrity, so the monitoring of preloading force during the winding construction is very important to PWOPF. After the winding construction, if the value of PWOPF winding preloading force is too low, the partition bumpy-ridge surface may easily slit or offset during the working process of press, which will influence the working performance and service life of press. If the value of winding preloading force is too high, it may easily result in the oversize deformation of PWOPF which will influence the subsequent installation of important components such as upper and lower internal bearing beams and the like.

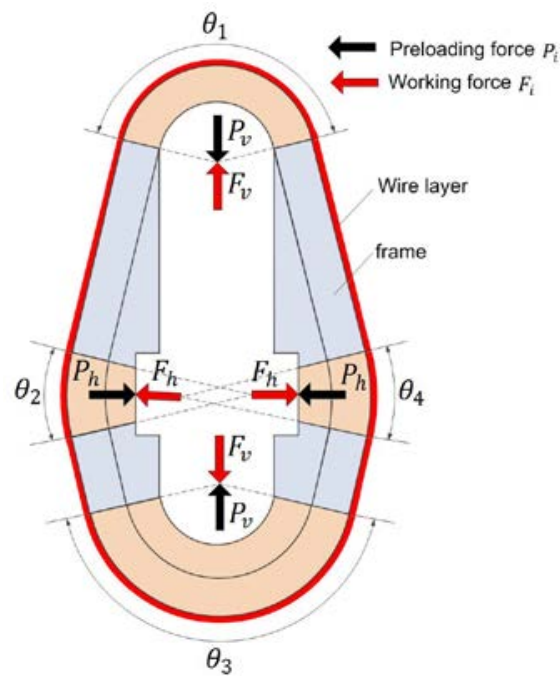

Fig. 1 Pre-stressed wire wound orthogonal preload frame structure

In the aspect of testing the preloading force of ordinary die forging hydraulic press, Peng Junbin ${ }^{[10]}$ et al developed the monitoring of preloading force of $360 \mathrm{MN}$ thick-wall steel pipe vertical extrusion press. Zhang Haoran ${ }^{[11]}$ et al developed the measurement of 1:15 model machine of 250/60 MN extrusion press. Liu Haixia ${ }^{[12]}$ et al developed the winding prestress test of the 1:10 model of 400 MN aviation die forging hydraulic press. Such new bearing structure as PWOPF is applied to the multi-ram die forging hydraulic press, it column consists of two segments of oblique columns with variable cross-sections and a segment of arched beam. Compared with ordinary preloading frame, the winding process should apply the preloading force from horizontal and vertical directions simultaneously, and the frame surface stress field distribution is complex. The unidirectional stress area is the key position for monitoring the preloading force during the wire winding process and is the direct reflection of applying actual preloading force on frame. PWOPF is not like the ordinary die forging hydraulic press with obvious unidirectional stress area of column, but it is difficult to select the unidirectional stress area, and it is difficult to test the preloading force.

In consideration of high experimental cost and long manufacturing cycle of heavy multi-ram die forging hydraulic press, in this paper, it takes $4 \mathrm{MN}$ multi-ram die forging hydraulic press for example, using the finite element temperature drop method to simulate the winding and preloading process of PWOPF, analyzing the characteristics of stress field distribution of its components and the unidirectional stress area, developing a reasonable preloading force test scheme and analyzing the integral rigidity during the preloading process of PWOPF as actually required by the project.

\section{Establishment of Finite Element Calculation Model}

Steel Wire Layer Temperature Drop Method. During the winding process, the steel wire layer has not changed the direction at the linear segment of outer contour of PWOPF and did not generate preloading force, but generating preloading force at the arc segment of the outer contour because of changing direction. At the time of finite element analysis, the equivalent surface pressure modeling method adopted by the steel wire layer is just on the basis of above characteristics. The specific equivalent surface pressure method includes the steps as follows: removing steel wire layer from the finite element calculation model; applying the preloading force applied by the steel wire layer on 
frame to the arc segment in the form of equivalent and uniformly distributed surface pressure $q_{i}$, neglecting the influence of steel wire layer on the linear segment of frame outer contour. The equivalent surface pressure value $q_{i}$ can be obtained by formula (1). As follow

$$
q_{i}=\frac{T}{R_{i} S}=\frac{1}{2} \frac{F_{i}}{R_{i} S} \frac{\eta}{\sin \frac{\theta_{i}}{2}}
$$

where $T$ is the tension of steel wire layer, $R_{i}$ is the radius of frame arc segment, $S$ is the width of steel wire slot, $\theta_{i}$ is the central angle of arc segment, and $\eta$ is the preloading coefficient. The equivalent surface pressure method neglects the overall rigidity increasing effect on the frame by the steel wire layer, the value of rigidity ratio is higher, which means the preloading force actually applied to the frame after winding preloading is higher than the finite element calculation value. In addition, the equivalent surface pressure method neglects the influence of the fractional force between the steel wire layer and the preload component during the finite element calculation.

In this paper, it proposes to simulate the winding and preloading process of PWOPF by the heat-expansion and cold-contraction characteristic of steel wire layer, namely the temperature drop method. The fundamental theory of steel wire layer temperature drop method is that: modeling the steel wire layer integrally by finite element; using the physical property of heat-expansion and cold-contraction of steel wire layer to predefine its temperature under the original state of calculation; reducing the temperature of steel wire layer gradually during the loading process, so as to apply certain temperature load on the steel wire layer and make the steel wire layer shrink and further generate expected preloading force towards PWOPF. This method has the advantages of replacing the calculation error caused by approximate estimation of surface pressure in the equivalent surface pressure method, not neglecting the effect of steel wire layer and being more identical with the actual winding and preloading process of the steel wire layer.

Establishment of Finite Element Model. 4 MN multi-ram die forging hydraulic press adopts two PWOPFs as its bearing structure. A single PWOPF ( as shown in figure 2) adopts Q345 welded-steel plate construction, with the external dimension of $2427 \mathrm{~mm} \times 1364 \mathrm{~mm} \times 221 \mathrm{~mm}$, the preloading coefficient of 2.0, the vertical bearing capacity of $2 \mathrm{MN}$, the horizontal bearing capacity of $0.5 \mathrm{MN}$, and the material of winding wires of $65 \mathrm{Mn}$. The material elasticity modulus is $E=2.09 \times 10^{5} \mathrm{MPa}$, the Poisson ratio is $v=0.3$; and the expansion factor of the steel wire layer is $\alpha=1.13 \times 10^{-5} \mathrm{~K}^{-1}$. According to the PWOPF loading and the symmetry of structure, it takes its one-half geometric model for the finite element analysis.

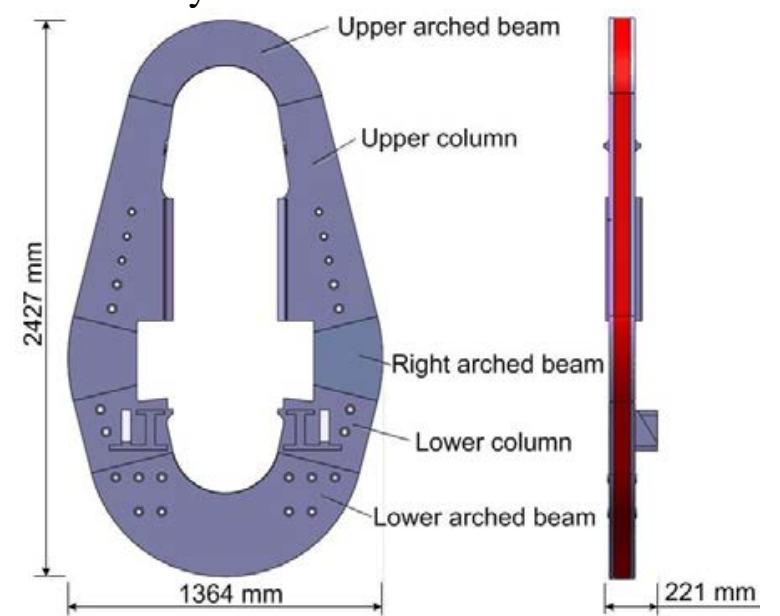

Fig. $24 \mathrm{MN}$ multi-ram die forging hydraulic press bearing frame

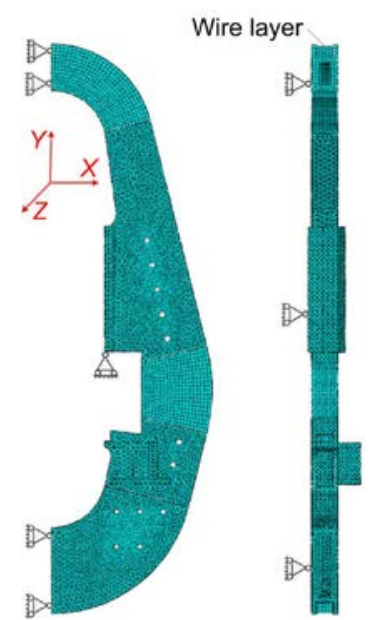

Fig. 3 Finite element model

The temperature of steel wire layer is set as $0 \mathrm{~K}$ under the initial state of original state of finite element calculation. It sets certain temperature drop value $(-150 \mathrm{~K})$ for trial computation in the first loading step, and it performs the temperature correction according to the difference between the preloading force obtained by PWOPF column calculation and the theoretical design value. Because of the approximately linear proportional relation between the temperature drop value and the column preloading force obtained by the finite element calculation, it can obtain the reasonable temperature 
drop value of $-370 \mathrm{~K}$ just by one correction. $4 \mathrm{MN}$ multi-ram die forging hydraulic press is dispersed by C3D10 tetrahedron element and C3D20R hexahedral element with good adaptability, and the finite element model is as shown in figure 3.

\section{Finite Element Analysis}

Stress Distribution Characteristics. Local cylindrical coordinates are respectively established for the upper, right and lower arched beams at PWOPF, the radial stress $S_{11}$ distributions of three components under this coordinate system are as shown in figure 4.

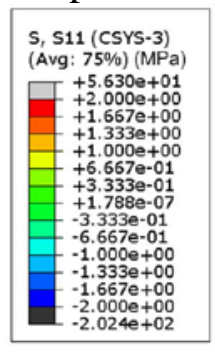

(a) Upper arched beam

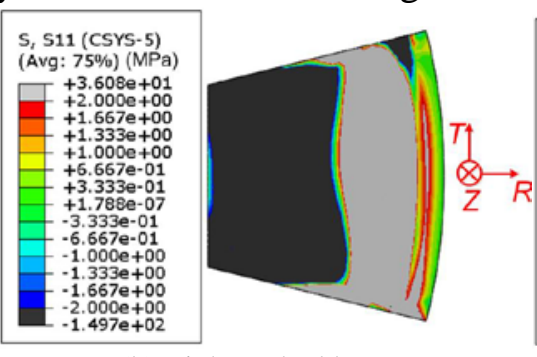

(b) Right arched beam

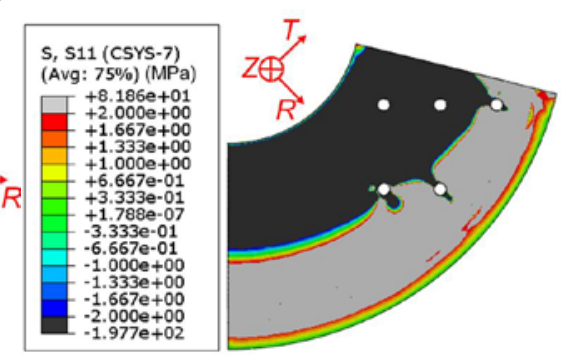

(c) Lower arched beam

Fig. 4 Stress distribution of arched beams

From figure 4, we can know that the radial stress distribution of each arch beam has the characteristics as follows: the external radial stress of each arched beam is mainly tensile stress, while the internal is mainly compressive stress; the tensile stress of each arched beam is reduced gradually from the outer edge along the radial direction and finally becomes compressive stress. The preloading force of steel wire layer is directly applied on the arc surfaces of steel wire slots of various arched beams during the winding process, as the steel wire winding layers increase, tons of preloading pressure results in the trend of inward contraction of arched beams, and their outer edges restrain the inward contraction, therefore, the radial stress near the center of circle shows compressive stress, and the radial stress near the edge shows tensile stress. Although the outer edges of arched beams have low radial stress values $\left(S_{11}=-2 \sim 2 \mathrm{MPa}\right)$ along the circumferential direction, it pays attention to the equivalent preloading force on the radial section during the PWOPF winding and preloading process, so it is not fit for sticking measurement.
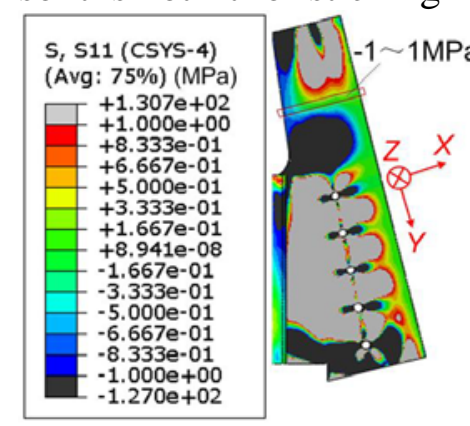

(a) Upper right column

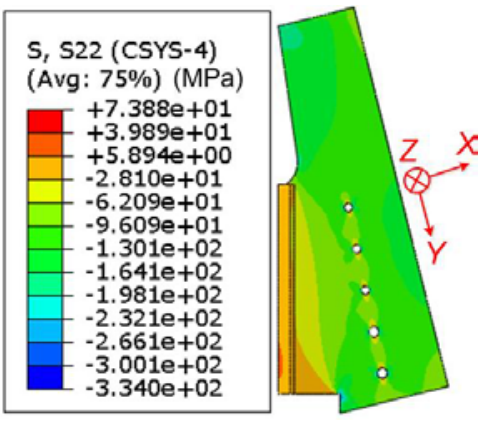

Fig. 5 Stress distribution of upper and lower columns

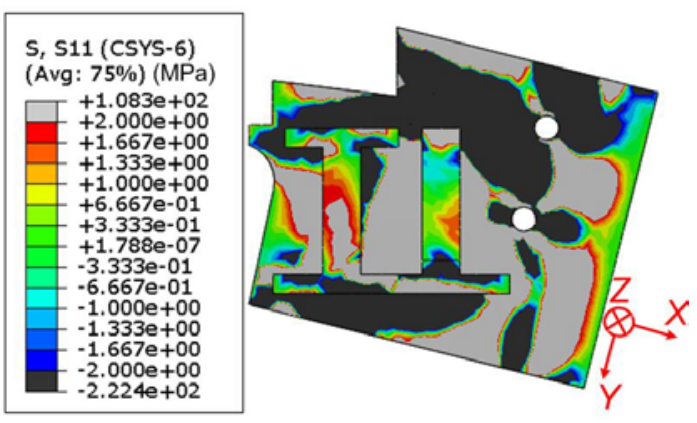

(b) Lower right column

It respectively establishes local rectangular coordinate systems for the upper right and lower right columns of PWOPF, and the stresses of two components under these coordinate systems are as shown in figure 5. From figure 5(a), we can know that the stress value along $X$ direction within the area indicated in the figure of upper right column is $S_{11}=-1 \sim 1 \mathrm{MPa}$, and the stress value along $Y$ direction within such area is $S_{22}=-110 \sim-150 \mathrm{MPa}$, it can be approximated as unidirectional stress area, and it is fit for sticking measurement of the actually applied preloading force during the PWOPF winding process. From figure 5(b), we can know that the distribution of the area with low stress value along $X$ direction of lower right column is complex and has no obvious characteristic, and it could not find out the section along $Y$ direction used for the sticking measurement of the preloading force of column.

The finite element result shows that there are approximate unidirectional stress areas at the outer edge of PWOPF arched beam and partial area at the top of column, but the unidirectional stress area of the outer edge of arched beam is distributed circumferentially, which is not fit for measuring the 
preloading force of the section. Therefore, it can select the section within the unidirectional stress area at the top of upper column to carry out actual sticking measurement of the preloading force during the PWOPF wire winding process.

Deformation Analysis. The winding and preloading process is the most dangerous state during the actual working condition of PWOPF structure, and the oversize deformation of bulk material of frame may easily occur as the increase of number of steel wire layers under the ultra-strong wire winding tension action, which results in structural damage and adverse effect on the subsequent installation of important components such as upper and lower bearing beams and the like. During the preloading process, as the number of steel wire layers increases gradually, the overall PWOPF will show inward shrink deformation. This deformation is the direct reflection of integral rigidity during the winding and preloading process of PWOPF, so it respectively takes points $a, b$ and $d$ as objects of study, for analyzing the overall deformation of the frame in vertical direction and horizontal direction during the winding and preloading process.

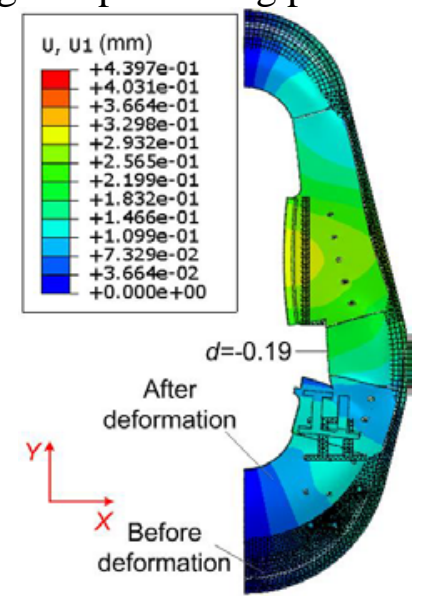

(a) Displacement in $X$ direction

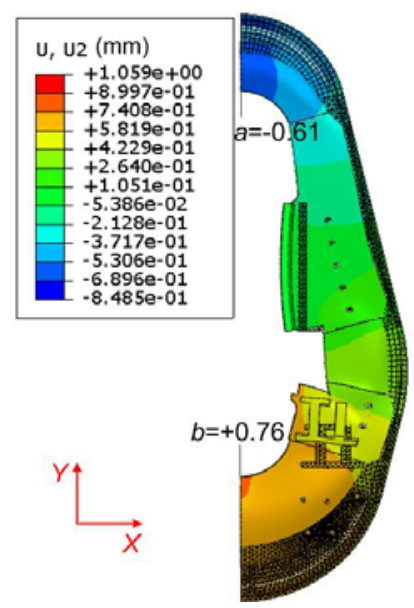

(b) Displacement in $Y$ direction

Fig. 6 PWOPF displacement distribution (Figure enlarged 200 times)

From figure 6(a), we can know that the maximum deflection value of PWOPF in $X$ direction within the range of overall length after winding and preloading is $+0.4397 \mathrm{~mm}$, and the maximum deflection variation in $X$ direction is $0.4397 \mathrm{~mm} / 2.427 \mathrm{~m}=0.181 \mathrm{~mm} / \mathrm{m}$. The amount of compression of PWOPF in $Y$ direction (the variation of displacement between points $a$ and $b$ ) is $-0.61 \mathrm{~mm}-(+0.76 \mathrm{~mm})=-1.37 \mathrm{~mm}$; according to the symmetry in $X$ direction, the variation of open-file size at the position of point $\mathrm{d}$ in $X$ direction (double of variation of displacement at the point $d$ ) is $20 .(19 \mathrm{~mm})=-0.38 \mathrm{~mm}$. We can know that after winding and preloading, the overall deformation of PWOPF in $X$ and $Y$ directions is less than it actual size, with better rigidity, which has little effect on the installation of upper and lower bearing beams and the guide processing and installation of moving beam and can meet the design requirement, and the above deformation values are of great importance in judging whether the PWOPF will slit or offset under the working load.

\section{Experimental Verification}

Winding Tension Design and Measurement of Steel Wire Layer. According to the design, it uses the A-type variable-tension wire winding technology for the $4 \mathrm{MN}$ multi-ram die forging bearing frame.

During the design of pre-stressed frame wire winding technology, the general calculating formula for the winding tension applied by each layer of steel wires can be got ${ }^{[13]}$.From the calculation, it obtains that the accumulated preloading force of single column after winding construction is 2.11 MN.

The stress test method used most frequently in project is resistance strain gauge electrical method, which means using the common resistance strain gauge and strain meter for measuring strain ${ }^{[14]}$. During the winding process (shown in figure 7), it measures the preloading force applied by PWOPF 
by DH3816 static strain measuring system and the resistance strain gauge once for winding every 3 layers.

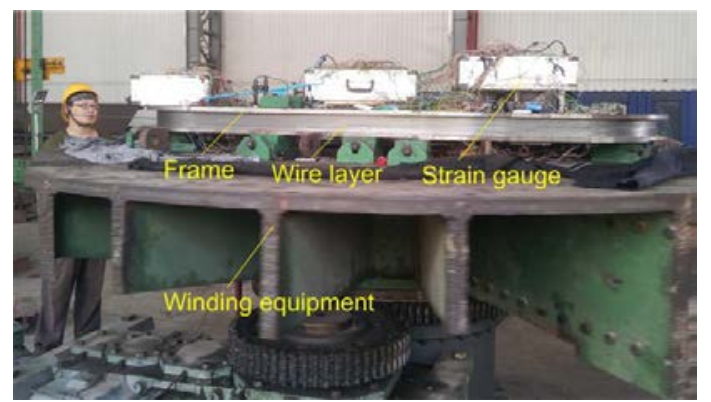

Fig. 7 Construction site of winding by $4 \mathrm{MN}$ multi-ram die forging press

The resistance strain gauge is made of metal material, and it resistance value changes along with the temperature change. It requires several days to finish the frame winding process of heavy multi-ram die forging hydraulic press, and due to the influences of diurnal temperature difference and other factors, it may easily cause the resistance variation of the resistance card during the measurement and influence the measurement accuracy, so it requires considering the problem of temperature compensation.

The common temperature compensation methods include patch compensation and self-compensation method. The patch compensation means sticking temperature compensation strain gauge on the independent test block with the same material as the frame and accessing the bridge circuit adjacent to the working strain gauge, so as to carry out temperature compensation. The independent test block does not feel the load strain and is positioned in the environment with the same temperature as the frame. The self-compensation method means arranging two working strain gauge at the frame vertically and closely, accessing the adjacent bridge circuit, also taking effect of compensating temperature mutually, and it's unnecessary to access specialized temperature compensation strain gauge. The self-compensation method is widely applied in the unidirectional stress state, and the compensation strain gauge should be close to the working strain gauge to the greatest extent. The compensation strain gauge and the working strain gauge should be arranged along the direction with lowest stress gradient. The actual test shows that the self-compensation method has the best measurement effect and can get satisfactory compensation precision ${ }^{[13]}$.

The finite element result shows that the partial area at the top of PWOPF column is approximated as unidirectional stress area. It selects key measuring section within this area and uses the self-compensation method for monitoring the preloading force, and the sticking mode is shown in figure 8(a). The PWOPF ensures the integrity of the frame by the winding and preloading force on various partition bumpy-ridge surfaces. The monitoring of preloading force on the partition bumpy-ridge surface is very important, but the finite element result shows that various partition bumpy-ridge surfaces are non-unidirectional stress area, and it pays attention to the normal force in the vertical direction of the partition bumpy-ridge surface during the winding process. Therefore, it may carry out unidirectional stress measurement in its normal direction and improves its precision by the patch temperature compensation, and the sticking mode is shown in figure 8(b).

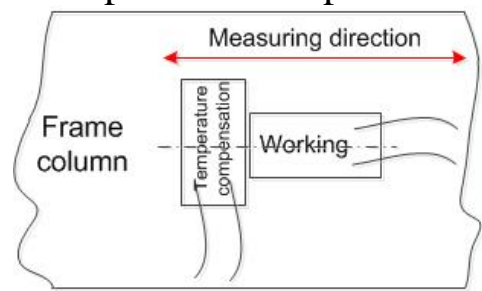

(a) Temperature self-compensation

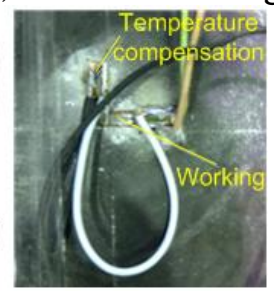

Fig. 8 Sticking mode of resistance strain gauge

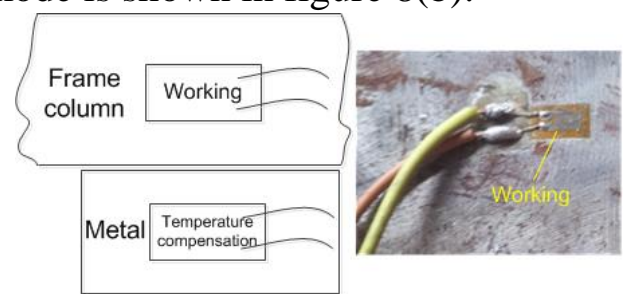

(b) Patch compensation

Selected measuring sections (section 1, section 2.....) and the sticking positions of resistance strain gauge on various sections are shown in figure 9. During the winding process, it measures the deformation of PWOPF by dial indicator, and measuring positions of dial indicator are shown in figure 9 (point $a \#$, point $b \# . . . .$. ). 


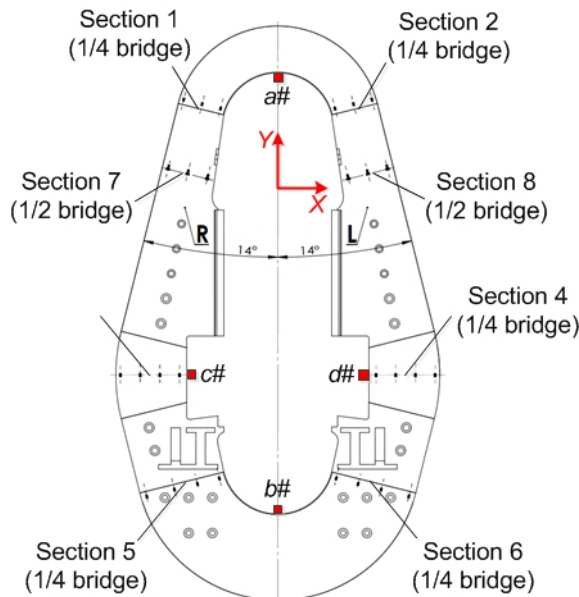

(a) Pre-stressed measuring sections

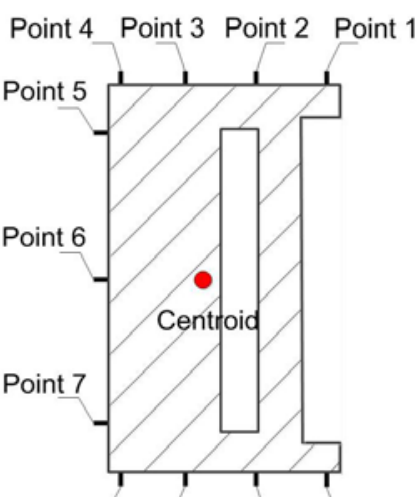

Point 8 Point 9 Point 10 Point 11

(b) Strain gauge sticking positions

Fig. 9 Schematic diagram of pre-stressed measurement

Result Discussion. During the winding process, as the steel wire winding layers increase, the correlation curve of the deformation values between points $a$ and $b$ and between points $c$ and $d$ of PWOPF actually measured by dial indicator and the values calculated by the finite element temperature drop method is shown in figure 10.

From figure 10, we can know that as the steel wire layers increase during the winding process, the curves of deformation values both between points $a$ and $b$ and between points $c$ and $d$ show the trend of increase, which means the overall deformation of PWOPF shows the inward shrink trend. As the steel wire layers increase, the curves the finite element calculation values of deformations between points $a$ and $b$ and between points $c$ and $d$ are almost straight lines, the curves of measured values of deformations between points $a$ and $b$ and between points $c$ and $d$ shows the tread of fast increasing early and slowly increasing lately along with the increase of the steel wire layers, and the measured deformation values during the whole winding process are all higher than the finite element calculation values. Mainly because there are gaps between the partitions of various components of PWOPF during the configuration process before winding, at the beginning of winding, the reading of dial indicator increases sharply because of gaps, and the reading of dial indicator shows the trend of increasing slows when the gaps disappear along with winding. The existence of gaps makes the measured deformation value of PWOPF higher, and it assumes no gaps between the partitions during finite element modeling, so the deformation values actually measured by dial indicator are all higher than the values calculated by finite element temperature drop method.

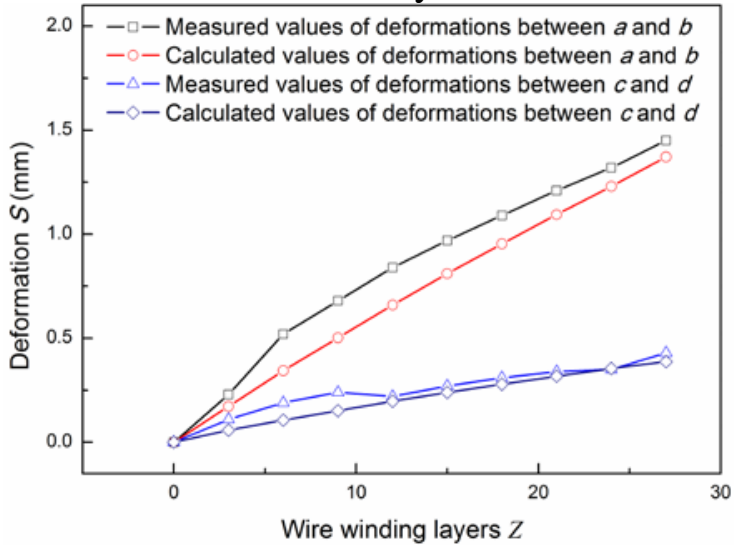

Fig. 10 Deformation curves of PWOPF

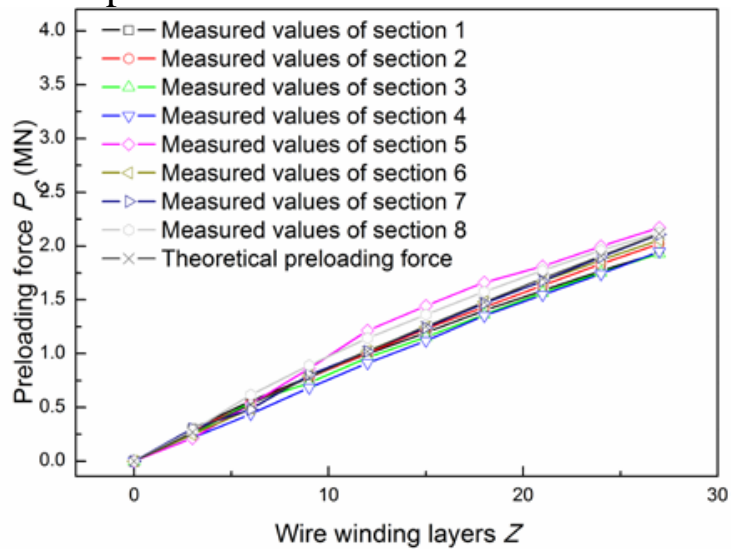

Fig. 11 Correlation curves of preloading Force

Figure 11 is, as the steel wire winding layers increase, the correlation curve of actual preloading force of PWOPF measured by resistance strain gauge and the designed theoretical preloading force. From figure 11, we can know that, as the steel wire winding layers increase, the measured values of preloading force of various sections are relatively identical with the designed theoretical values, both showing the gradually increasing trend. During the wire winding process, as long as the winding and preloading state is under safe state, the whole frame is always in the elastic deformation stage, therefore, as the steel wire winding layers increase, the preloading force of column should also have 
good linear relation ${ }^{[10]}$. The preloading force of various measuring sections of $4 \mathrm{MN}$ multi-ram die forging PWOPF has better linearity along with the increase of steel wire layers, and the measured data have high reliability.

From figure 10, we can know that the finite element calculation values of deformations between points $a$ and $b$ and between points $c$ and $d$ of PWOPF after wire winding and preloading and the values actually measured by dial indicator are shown in table 1.

Table 1 Comparision of measured deformation values and calculated values of PWOPF

\begin{tabular}{|c|c|c|c|c|}
\hline $\begin{array}{c}\text { Measuring } \\
\text { point }\end{array}$ & $\begin{array}{l}\text { Deformation } \\
U(\mathrm{~mm})\end{array}$ & $\begin{array}{l}\text { Measured Deformation Value } \\
\qquad \Delta U(\mathrm{~mm})\end{array}$ & $\begin{array}{l}\text { Finite Element Calculation Value } \\
(\mathrm{mm})\end{array}$ & $\begin{array}{c}\text { Relative Error } \\
(\%)\end{array}$ \\
\hline$a$ & +0.91 & \multirow{2}{*}{$\Delta U_{a b}=1.45$} & \multirow{2}{*}{1.37128} & \multirow{2}{*}{$5.4 \%$} \\
\hline$b$ & +0.54 & & & \\
\hline C & +0.28 & \multirow{2}{*}{$\Delta U_{c d}=0.43$} & \multirow{2}{*}{0.38716} & \multirow{2}{*}{$9.9 \%$} \\
\hline$d$ & +0.15 & & & \\
\hline
\end{tabular}

From table 1, we can know that the maximum absolute relative error of the measured values of deformations between points $a$ and $b$ and between points $c$ and $d$ of PWOPF after wire winding and preloading and the finite element calculation values is $9.9 \%$, and the minimum absolute relative error is $5.4 \%$. From the point of view of practical engineering application, this maximum error value is absolutely acceptable, and it shows the feasibility of simulating the wire winding process of PWOPF by finite element temperature drop method.

Table 2 Comparison of preloading force of various sections $(Z=27)$

\begin{tabular}{cccc}
\hline Measuring section & $\begin{array}{c}\text { Measured preloading force } \\
(\mathrm{MN})\end{array}$ & $\begin{array}{c}\text { Theoretical preloading force } \\
(\mathrm{MN})\end{array}$ & \multicolumn{2}{c}{$\begin{array}{c}\text { Relative error } \\
(\%)\end{array}$} \\
\hline Section 1 & 1.9365 & & $-8.4 \%$ \\
Section 2 & 2.0206 & & $-4.4 \%$ \\
Section 3 & 1.9305 & $-8.7 \%$ \\
Section 4 & 1.9489 & $-7.8 \%$ \\
Section 5 & 2.1703 & 2.1135 & $+2.7 \%$ \\
Section 6 & 2.0560 & & $-2.7 \%$ \\
Section 7 & 2.1078 & $-0.3 \%$ \\
Section 8 & 2.1257 & $+0.6 \%$ \\
Mean Value of Sections & 2.0370 & $-3.6 \%$ \\
\hline
\end{tabular}

Table 2 is the comparison between the measured values and designed theoretical values of preloading force of various sections after finishing 27 layers of steel wire layer winding. We can know that after finishing winding the 27th layer of steel wires for PWOPF, the section of column with the maximum error of preloading force is the symmetrical section of left arched beam, and the actual winding and preloading force is $8.7 \%$ less than the theoretical value. The section of column with the minimum error of preloading force is the section within the unidirectional stress area of the upper left column, and the actual winding and preloading force is $0.3 \%$ less than the theoretical value. The average actual winding and preloading force of various sections of the whole PWOPF column is 3.6\% less than the theoretical value.

During the winding process of PWOPF, the influence of actual winding error and measurement error will necessarily and inevitably affect the measured preloading force of column. Therefore, from the perspective of analysis of engineering application, the relative error of average winding and preloading force of whole PWOPF column 3.6\% is acceptable, and accordingly, the method of measuring the preloading force of PWOPF by sticking scheme developed in this paper on the basis of finite element temperature drop method is feasible and can meet the actual engineering application.

\section{Conclusions}

From the analysis of comparison between the PWOPF deformation values obtained by finite element temperature drop method and the measured values by $4 \mathrm{MN}$ multi-ram die forging hydraulic press test, we can know that maximum relative error between the result tested by the dial indicator and the finite element calculation result is $9.9 \%$, and considering the influence of actual winding error and measurement error during the winding process, this result is acceptable in engineering. Therefore, it is feasible to simulate the wiring winding process of PWOPF by the finite element steel wire temperature drop method. 
The finite element result shows that the partial area at the top of upper column of PWOPF is approximated as unidirectional stress area. It can select the section within this area to use the self-compensation sticking mode for measuring the preloading force. The preloading force on various partition bumpy-ridge surfaces of the PWOPF are the key surfaces ensuring its integrity. Because the stress distribution within this area is non-unidirectional stress area, it measures the preloading force by the patch temperature compensation method.

After wire winding, the preloading force result obtained by testing various sections is fundamentally identical with the theoretical design value, the relative error of the preloading forces measured for the sections within the unidirectional stress area at the top of upper column are all less than $1 \%$. This area is the key area for monitoring the preloading force of PWOPF, and it shows that the PWOPF construction quality meets the design requirement and proves that the sticking scheme developed on the basis of finite element temperature drop method is feasible. This preloading force measuring method provides basis for the quality detection and evaluation during the process of PWOPF winding technology and solves the engineering problem of PWOPF stress test during the process of designing and manufacturing heavy multi-ram die forging press.

\section{Funding}

The authors would like to acknowledge the funding of National Science and Technology Major Project of China, No.2012ZX04010082.

\section{References}

[1] Shixuan Han, Multi-ram Forge, 1th ed., Shanghai People’s Press, Shanghai, 1977.

[2] Zongtang Lin, Study on Heavy Prestressed Concrete Hydraulic Press, 1th ed., Tsinghua University Press, Beijing, 1988.

[3] Lan Ji, Introduction of foreign multi-ram and split die forge, J. Forge Machinery. 3 (1973) 39-53.

[4] Xi'an Institute of Heavy Machinery Room One, Introduction of multi-way forge press development in foreign country, J. Heavy Machinery. 3 (1977) 80-84.

[5] Fengchun Zhi, Yunxiu Xie, Xianwen Liu, Multi-way forge equipment in foreign country, J. Cfhi Technology. 4 (1995) 1-15.

[6] Dengguo Xiong, 10000 tons multi-ram forge hydraulic press, J. Heavy Machinery. 1 (1978) 42-50.

[7] Shouning GUO, Analysis and treatment for lacking of preload of the 10000 tons multi-way forge hydraulic press horizontal frame, J. Heavy Machinery. 11 (1981) 47-61.

[8] Fen Lin, Lei Zhang, Fu Sun, et al, Multi-ram forge process and its equipment development, J. Journal of Mechanical Engineering. 48(1) (2012) 1-8.

[9] Changyong Liu, Renji Zhang, Yongnian Yan, et al, Thermal stress analysis on wire winded subdivided-combined larger extrusion container, J. Engineering Mechanics. 28(5) (2011) 207-211.

[10] Junbin Peng, Pre-stressed bumpy ridge joining method for mechanical structure, D. Tsinghua University, Beijing, 2008.

[11] Haoran Zhang, Research on 250/60MN steel tube extrusion press with single frame \& double wrapping structure, D. Tsinghua University, Beijing, 2009.

[12] Haixia Liu, Yongnian Yan, Pan Zeng, et al, Pillar and arched girder totally bumpy ridge joining frame with steel wire wound, J. Journal of Mechanical Engineering. 47(4) (2011) 82-87.

[13] Yongnian Yan, Xinlu Yu, Prestressed Structure in Mechanical Design, 1th ed., China Machine Press, Beijing, 1989.

[14] Xiuyuan Zheng, Daji Xie, Measuring Technology on Stress and Strain, 1th ed., Defense Industry Press, Beijing, 1985. 